Ex. 3. The principle may be of use in cases not coming under the above theorem. For example, the simple elementary identity

$$
\left(x^{2}+x y+y^{2}\right)\left(x^{2}-x y+y^{2}\right)=x^{4}+x^{2} y^{2}+y^{4}
$$

may be written

$$
\left(\Sigma x^{2}+\Sigma x y\right)\left(\Sigma x^{2}-\Sigma x y\right)=\Sigma x^{4}+\Sigma x^{2} y^{2},
$$

for two letters.

For three letters, we deduce easily by the above method

$$
\left(\Sigma x^{2}+\Sigma x y\right)\left(\Sigma x^{2}-\Sigma x y\right)=\Sigma x^{4}+\Sigma x^{2} y^{2}-2 \Sigma x \Sigma x y z,
$$

the coefficient of the last term being determined by putting each letter equal to 1 .

This again gives for four letters

$$
\begin{aligned}
\left(\Sigma x^{2}+\Sigma x y\right) & \left(\Sigma x^{2}-\Sigma x y\right) \\
=\Sigma x^{4}+\Sigma x^{2} y^{2}-2 \Sigma x \Sigma x y z+2 \Sigma x y z u, &
\end{aligned}
$$

which is now true for any number of letters.

John Dougall.

\title{
A physical solution of the Apollonian problem.
}

This celebrated problem-to describe a circle to touch three given circles-admits of a simple experimental solution which might be found interesting by a mathematical class.

The surface on which the circles lie may be either a plane or a sphere, but the latter case is the more convenient to deal with, and will be the one taken.

We suppose also that the points of intersection of the circles are all real-though this is not essential-so that eight triangles are formed, the sides of which are circular arcs. The circles are to be fitted together in the form of wires or thin strips of wood.

Now take a spherical ball of any (not too small) size, and drop it. into the opening formed within one of the triangles $A B C$, so that it meets the ares $B C, C A, A B$ at $X, Y, Z$ respectively. Then the circle through the points $X, Y, Z$ is one of the circles touching the three given circles.

Proof. The points $X, Y, Z$ lie on both spheres, and the circle $X Y Z$ is therefore the common section of the spheres. The circles 
$B X C$ and $X Y Z$ lie on the same sphere; they meet at $X$, but they cannot meet again, since the circle $B X C$ meets the spherical ball, on which the circle $X Y Z$ lies, at one point only; the circle $X Y Z$ therefore touches $B X C$; and similarly it touches $C Y A$ and $A Z B$.

Instead of a spherical ball a plane, in the form of a flat board, may be used.

Using the flat board, we can even extend the construction to the case when three plane sections of a quadric, say of an ellipsoid, are taken instead of three circles on a sphere. It easily proved that through three ellipses lying in different planes, so that any two of them have two common points, a quadric can be drawn. ${ }^{1}$

If the board is brought down on three such ellipses, realised in pieces of wire properly fastened together, the common section of the plane and quadric will touch the three ellipses; in other words, a conic can be drawn through the three points where the plane meets the three ellipses so as to touch them at these points; the proof is practically the same as before.

An ellipsoidal ball would not serve in this case. Its section by the plane $X Y Z$ would certainly meet each of the three ellipses at one point only, but there is no guarantee that that section is identical with the section of the given quadric by the plane $X Y Z$, so that meeting in one point only does not imply contact.

Dr John Dougall.

\section{A Note on Conjugate Permutations.}

$\S 1$. Two permutations of the natural order $(123 \ldots n)$ are said to be conjugate when each number and the number of the place in the one permutation are interchanged in the case of the other permutation.

For example (32541) and (52143) are conjugate permutations of (12345).

Conjugate permutations seem to have been first considered by H. A. Rothe ${ }^{2}$ in 1800 . They arise spontaneously, of course, when we

1 if. H. F. Baker, Principles of Geometry, Vol. III., p. 10.

2 See Muir, History, Part I., pp. 59-60. Netto (Combinatorik, p. 118) seems unaware of Rothe's work. 